\title{
Combined Inhibition of C5 and CD14 Attenuates Systemic Inflammation in a Piglet Model of Meconium Aspiration Syndrome
}

\author{
Anub Mathew Thomas ${ }^{a}$ Camilla Schjalm $^{a}$ Per H. Nilsson ${ }^{a}$ b \\ Paal H.H. Lindenskov ${ }^{c}$ Runa Rørtveit ${ }^{d}$ Rønnaug Solberg ${ }^{e, f}$ \\ Ola Didrik Saugstad $^{\mathrm{e}}$ Magnus M. Berglund ${ }^{9}$ Patrik Strömberg ${ }^{g}$ \\ Corinna Lau ${ }^{\text {h }}$ Terje Espevik $^{i} \quad$ Johan Høgset Jansen ${ }^{d} \quad$ Albert Castellheim $^{j}$ \\ Tom Eirik Mollnes ${ }^{a, h, i, k} \quad$ Andreas Barratt-Due ${ }^{a, c}$ \\ a Department of Immunology, Oslo University Hospital and K.G. Jebsen IRC, University of Oslo, Oslo, Norway; \\ ${ }^{\mathrm{b}}$ Linnaeus Centre for Biomaterials Chemistry, Linnaeus University, Kalmar, Sweden; 'Division of Emergencies and \\ Critical Care, Oslo University Hospital, Oslo, Norway; ${ }^{\mathrm{d}}$ Faculty of Veterinary Medicine and Biosciences, Norwegian \\ University of Life Sciences, Oslo/Ås, Norway; ${ }^{\text {} D e p a r t m e n t ~ o f ~ P e d i a t r i c ~ R e s e a r c h, ~ O s l o ~ U n i v e r s i t y ~ H o s p i t a l, ~ U n i v e r s i t y ~}$ \\ of Oslo, Oslo, Norway; ${ }^{f}$ Institute of Surgical Research, Oslo University Hospital, Oslo, Norway; ${ }^{9}$ Research and

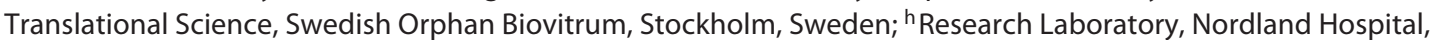 \\ Bodø, Norway; ${ }^{i}$ Center of Molecular Inflammatory Research and Department of Cancer Research and Molecular \\ Medicine, Norwegian University of Science and Technology, Trondheim, Norway; ${ }^{j}$ Department of Anaesthesiology \\ and Intensive Care, Institute of Clinical Sciences, Sahlgrenska Academy, University of Gothenburg, Gothenburg, \\ Sweden; ${ }^{k}$ K.G Jebsen TREC, University of Tromsø, Tromsø, Norway
}

\section{Keywords}

Meconium aspiration syndrome $\cdot \mathrm{C} 5$ - Complement . CD14 $\cdot$ Toll-like receptor $\cdot$ Innate immunity $\cdot$ Systemic inflammation

\begin{abstract}
Background: Meconium aspiration syndrome (MAS) is a severe lung condition affecting newborns and it can lead to a systemic inflammatory response. We previously documented complement activation and cytokine release in a piglet MAS model. Additionally, we showed ex vivo that meconium-induced inflammation was dependent on complement and Toll-like receptors. Objectives: To assess the efficacy of the combined inhibition of complement (C5) and CD14 on
\end{abstract}

\begin{tabular}{ll}
\hline KARGER & ( 2018 The Author(s) Karger \\
& Published by S. Karger AG, Basel Open access \\
E-Mail karger@karger.com & This article is licensed under the Creative Commons Attribution- \\
www.karger.com/neo & $\begin{array}{l}\text { NonCommercial-NoDerivatives 4.0 International License (CC BY- } \\
\text { NC-ND) (http://www.karger.com/Services/OpenAccessLicense). } \\
\text { Usage and distribution for commercial purposes as well as any dis- } \\
\text { tribution of modified material requires written permission. }\end{array}$
\end{tabular}

systemic inflammation induced in a forceful piglet MAS model. Methods: Thirty piglets were randomly allocated to a treatment group receiving the $\mathrm{C} 5$-inhibitor SOBI002 and anti-CD14 $(n=15)$ and a nontreated control group $(n=15)$. MAS was induced by intratracheal meconium instillation, and the piglets were observed for $5 \mathrm{~h}$. Complement, cytokines, and myeloperoxidase (MPO) were measured by ELISA. Results: SOBI002 ablated C5 activity and the formation of the terminal complement complex in vivo. The combined inhibition attenuated the inflammasome cytokines IL-1 $\beta$ and IL- 6 by $60(p=0.029)$ and $44 \%(p=0.01)$, respectively, and also MPO activity in the bronchoalveolar fluid by $42 \%$ ( $p=0.017)$. Ex vivo experiments in human blood revealed that the combined regimen attenuated meconium-induced MPO release by $64 \%(p=0.008)$, but there was only a negli-
Andreas Barratt-Due

Division of Emergencies and Critical Care

Oslo University Hospital Rikshospitalet

NO-0027 Oslo (Norway)

E-Mail andreas.barrattdue@gmail.com 
gible effect with single inhibition, indicating a synergic cross-talk between the key molecules C5 and CD14. Conclusion: Combined inhibition of $\mathrm{C} 5$ and $\mathrm{CD} 14$ attenuates meconium-induced inflammation in vivo and this could become a future therapeutic regimen for MAS.

(C) 2018 The Author(s)

Published by S. Karger AG, Basel

\section{Introduction}

Meconium aspiration syndrome (MAS) is a lung condition that affects newborn infants that inhale meconium prior to or during birth [1]. The pathophysiology is complex, involving a mechanical blockade of the airways, epithelial injury, pulmonary hypertension, surfactant inactivation, and inflammation. MAS reflects a severity spectrum that requires lung-supportive treatment ranging from oxygen delivery, the administration of surfactant, mechanical ventilation, and extra corporeal membrane oxygenation in critical cases [2]. Although the incidence of severe MAS is low, it is associated with high mortality (20-30\%) in low-income countries [3].

Meconium is a complex mixture of water, epithelial cells, bile, mucus and amniotic fluid, containing numerous substances and danger motifs that may induce inflammation [4]. Experimental studies have revealed that meconium induces a potent local inflammation in the lungs, with the features: leukocyte infiltration, alveolar macrophage activation, the release of myeloperoxidase (MPO), increased cytokine formation, and apoptosis [5]. Additionally, MAS-induced complement activation and cytokine release has been shown experimentally in piglets [6], and the systemic inflammatory response has been shown to correlate with the severity of MAS among neonates [7].

Two important recognition systems of innate immunity, the complement and the Toll-like receptor (TLR) systems, appear to play an important role in meconiuminduced inflammation. The degree of complement activation has previously been shown to reflect the severity of MAS [8]. In whole blood, meconium-induced inflammation is differentially attenuated by inhibition of either the complement or the accessory TLR molecule, CD14, but is abolished by their combined inhibition $[9,10]$. Although complement and TLRs act independently, they are connected through mutual interaction and extensive crosstalk, so a combined blockage of these branches represents a potential pronounced anti-inflammatory action on downstream inflammatory responses $[9,11]$.

The aim of this study was to examine the effect of a combined inhibition of complement (C5) and TLR
(CD14) in a piglet model of MAS. We hypothesized that this regimen would dampen the local and systemic inflammatory response evoked by meconium [1].

\section{Materials and Methods}

Meconium and Interventional Drugs

Meconium was prepared according to a previously described method [12], reconstituted to $99 \mathrm{mg} / \mathrm{mL}$ using PBS, and confirmed endotoxin-free. Complement inhibition was obtained by using the Affibody SOBI002, provided by Swedish Orphan Biovitrum, (Stockholm, Sweden). SOBI002 (molecular weight $12 \mathrm{kDa}$ ) consists of 2 protein domains, an Affibody ligand, targeting and preventing the cleavage of $\mathrm{C} 5$, which is fused to an albumin-binding domain that extends its half-life by binding plasma albumin [13]. Inhibition of CD14 was obtained by using a recombinant antiporcine CD14 IgG2/4 monoclonal antibody (clone rMIL2), and a recombinant anti-human CD14 IgG2/4 (clone r18D11), previously described in detail [14].

\section{Animals, Anesthesia, Exclusion Criteria, Surgery, and} Monitoring

Norwegian Landrace piglets (Sus scrofa domesticus) of either sex, 2 days old at the most, with a mean weight of $2 \mathrm{~kg}$ (range 1.8$2.3 \mathrm{~kg}$ ) were used. Housekeeping, anesthesia, surgery, euthanasia, and recording of hemodynamics and respiratory parameters were performed according to previously described procedures [15].

\section{Bronchoalveolavar Lavage}

The amount of saline used in the bronchoalveolavar lavage (BAL) procedure was tested in pilot experiments. Saline was inserted intratracheally followed by $10 \mathrm{~s}$ of manual ventilation, before BAL fluid (BALF) was obtained through suction and stored at $-70^{\circ} \mathrm{C}$. A volume of $5 \mathrm{~mL}$ of saline occasionally provided an insufficient sample amount, whereas $10 \mathrm{~mL}$ of saline was sufficient without affecting the lung function. Ten minutes after the BAL procedure with $10 \mathrm{~mL}$ of saline, the oxygen saturation, compliance and ventilation index were all at level similar to before the procedure. So $10 \mathrm{~mL}$ of saline was used in the study.

\section{Experimental in vivo Model of MAS}

Thirty piglets were randomly allocated into 2 groups, to which the investigators were blinded: a treatment group $(n=15)$ and a control group $(n=15)$. In addition, 5 sham animals were included (Fig. 1a). After surgery and stabilization, the BALF was sampled. The intervention was provided intravenously prior to meconium instillation; anti-CD14 as a bolus of $5 \mathrm{mg} / \mathrm{kg}$, but SOBI002 as a bolus of $5 \mathrm{mg} / \mathrm{kg}$ and then as a continuous infusion of $2.5 \mathrm{mg} / \mathrm{kg} / \mathrm{h}$ for the 5-h observational period. As a supplement to systemic intervention, anti-CD14 and SOBI002 were additionally provided locally in the lungs, (diluted with PBS to a final concentration of 300 and $150 \mu \mathrm{g} / \mathrm{mL}$, respectively) and mixed with the meconium solution when this was installed intratracheally in the treatment group. An equivalent amount of saline replaced the interventional drugs in both the control and sham groups. Hypoxia, mimicking perinatal asphyxia, was induced with $8 \%$ oxygen in nitrogen (AGA, Oslo, Norway) for $10 \mathrm{~min}$ and performed just before meconium instillation. Meconium $(5.5 \mathrm{~mL} / \mathrm{kg})$ was administered intratrache- 

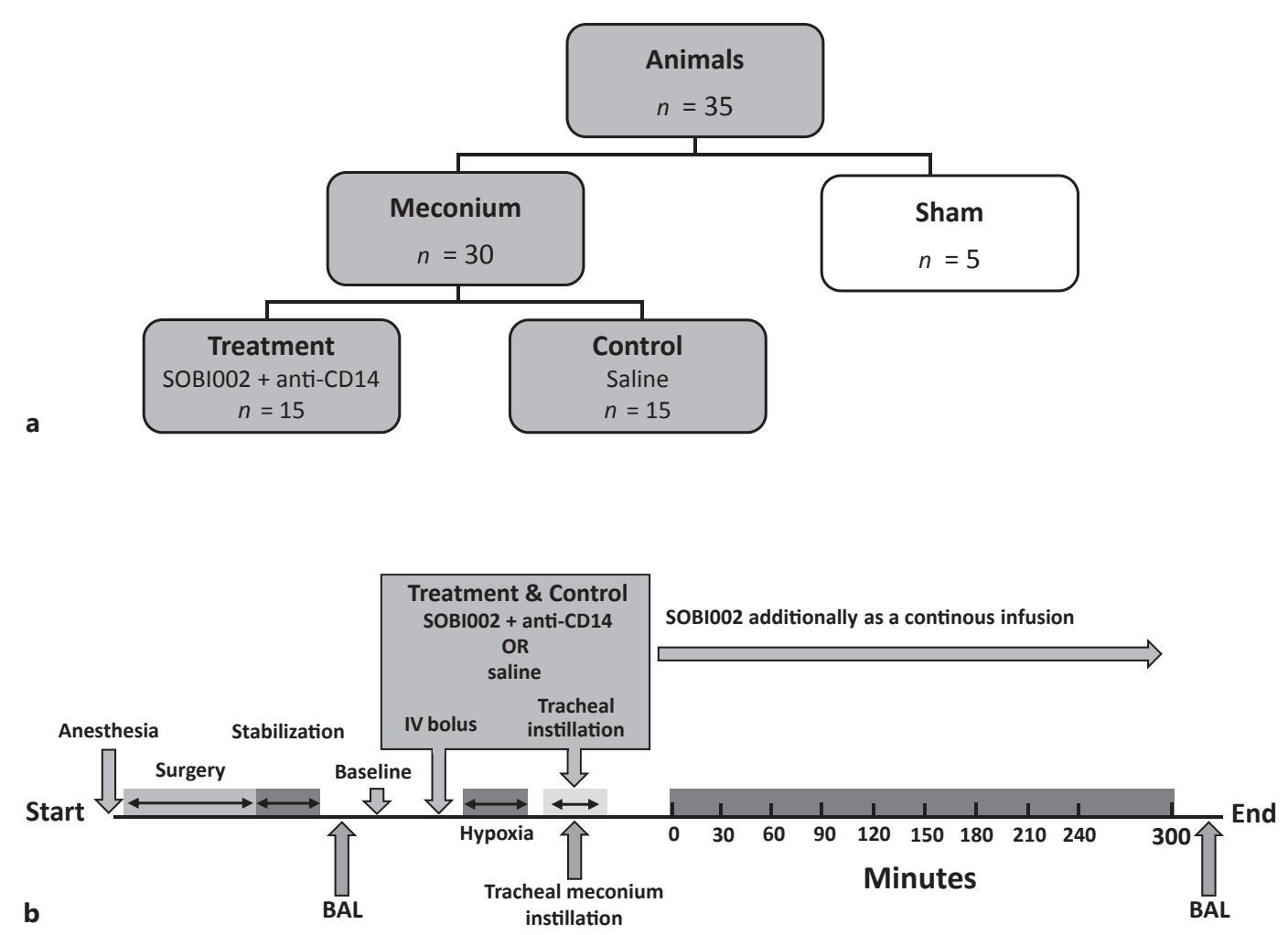

Fig. 1. Experimental design. a Thirty piglets were randomly allocated to a treatment group $(n=15)$ that received the complement (C5) inhibitor SOBI002 and anti-CD14 and a control group $(n=$ 15). Five animals were additionally included in a sham group. b Schematic illustration of the experimental setup. After surgery and stabilization, the intervention with anti-CD14 and SOBI002 was given as an intravenous (IV) bolus. SOBI002 was also given as a continuous infusion throughout the observational period. Hy-

ally in 4 boluses within $4 \mathrm{~min}$. The piglets were manually ventilated with $100 \%$ oxygen in between the boluses. Continuous reoxygenation by mechanical ventilation (Baby log 8000+; Dragerweck, Lübeck, Germany) to preserve an oxygen saturation of $>85 \%$, a $\mathrm{pCO}_{2}$ of $<8 \mathrm{kPa}$ limited by an upper peak inspiratory pressure of $40 \mathrm{~cm} \mathrm{H}_{2} \mathrm{O}$, a positive end expiratory pressure of $5 \mathrm{~cm} \mathrm{H}_{2} \mathrm{O}$, and a maximum respiratory frequency rate of $40 / \mathrm{min}$, according to a previously described procedure [12]. Blood samples were taken at regular time points, directly centrifuged, and put on ice before both EDTA-plasma and serum were stored at $-70^{\circ} \mathrm{C}$. BAL was again performed at the time of death (Fig. 1b).

\section{Experiments ex vivo of Meconium in Human Whole Blood}

The ex vivo human whole-blood model has previously been described in detail [16]. Briefly, fresh human whole blood was collected from healthy donors in vacutainer tubes anticoagulated with the specific thrombin inhibitor lepirudin (Refludan, Pharmion, Copenhagen, Denmark). Whole blood was preincubated with the poxia, mimicking perinatal asphyxia, lasted $10 \mathrm{~min}$ before meconium was installed in 4 separate boluses over $4 \mathrm{~min}$. As an additive to systemic intervention, SOBI002 and anti-CD14 were administered intratracheally, together with the instillation of meconium, reaching all meconium-affected regions of the lungs. An equivalent amount of saline replaced the interventional drugs in the control and sham groups. Bronchoalveolavar lavage (BAL) was performed before baseline and the end of the experiment. inhibitors SOBI002 at $300 \mu \mathrm{g} / \mathrm{mL}$ and/or anti-human CD14 IgG2/4 (r18D11) at $15 \mu \mathrm{g} / \mathrm{mL}$ and/or PBS for $5 \mathrm{~min}$ at $37^{\circ} \mathrm{C}$. Meconium $(1 \mathrm{mg} / \mathrm{mL})$ was then added and incubated for $30 \mathrm{~min}$ at $37^{\circ} \mathrm{C}$. Phorbol 12-myristate 13-acetate (100 ng/mL; Sigma-Aldrich, Darmstadt, Germany) was used as positive control. At the end of incubation, EDTA $(20 \mathrm{mM})$ was added to prevent additional complement activation, and the tubes were centrifuged for $15 \mathrm{~min}$ at $1,400 \mathrm{~g}$ at $4{ }^{\circ} \mathrm{C}$. Plasma was isolated and stored at $-70^{\circ} \mathrm{C}$ until analyzed.

\section{Enzyme Immunoassays}

The C5 inhibitory effect of SOBI002 was tested by using heataggregated immunoglobulin G (IgG) known to be a strong activator of the classical complement pathway [17]. Serum was added to microtiter plates coated with heat-aggregated IgG, and complement activation was measured as the formation of soluble (s)C5b9. The sC5b-9 terminal complement complex was measured in an enzyme immunoassay as previously described in detail [18]. The 


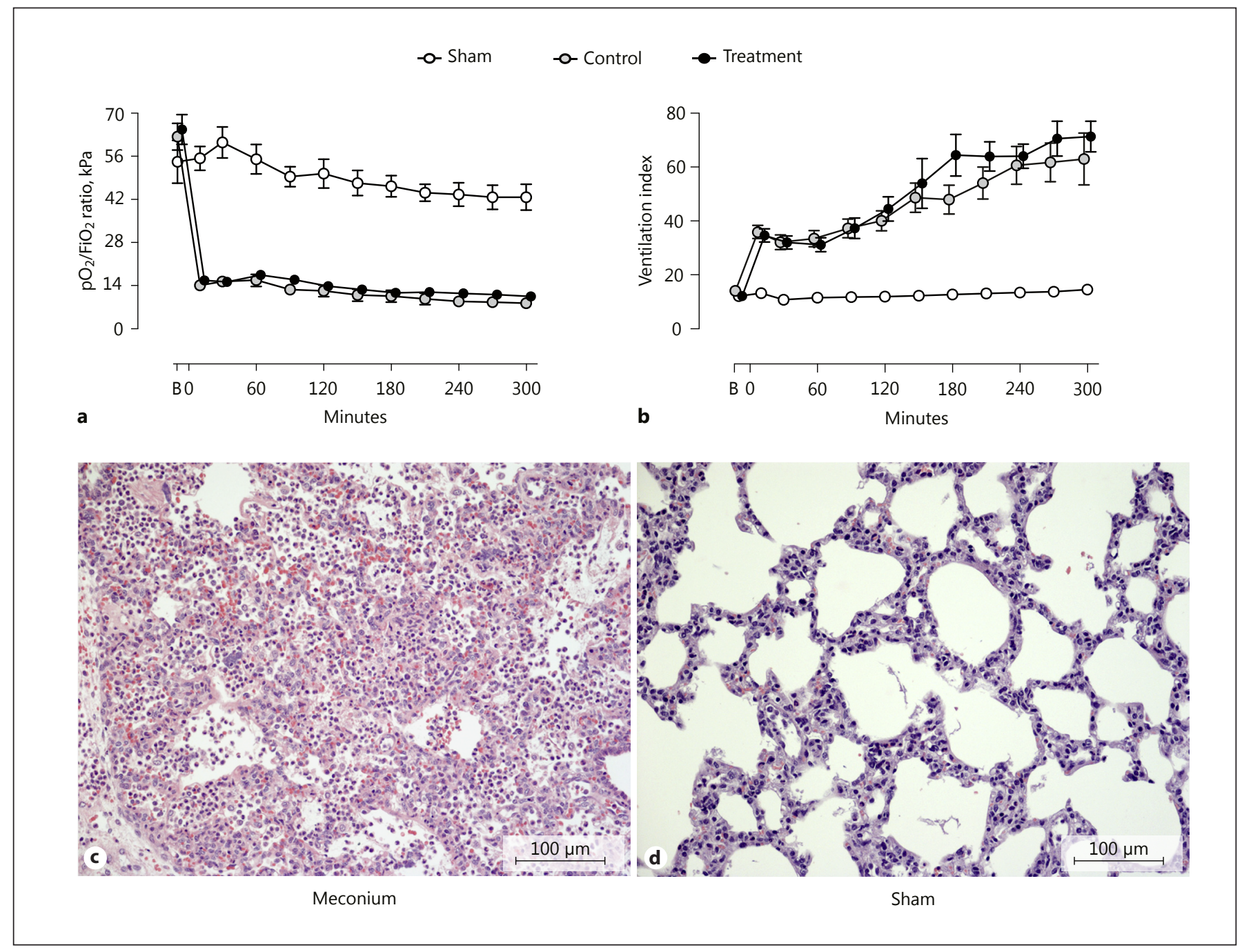

Fig. 2. Lung function and histopathology. Pulmonary function measured as $\mathrm{pO}_{2} / \mathrm{FiO}_{2}$ ratio (a) indicating hypoxemia, and ventilation index (b) reflecting the magnitude of ventilation support were measured at baseline, before instillation of meconium, and throughout the experiment. Lung necropsy specimens were collected after euthanasia, processed, and stained. One lung biopsy from a representative meconium treated animal (c) and 1 from a

cytokines TNF, IL-6, IL-1 $\beta$, and IL- 8 were analyzed by quantitative porcine immunoassay kits from R\&D Systems (Minneapolis, MN, USA). MPO activity in the porcine plasma was measured using the species independent MPO activity assay kit (Fluorometric, ab 11749; Abcam, Cambridge, UK). The MPO level in human plasma was quantified in the human-specific ELISA MPO kit HK 324 (Hycult Biotech, Uden, The Netherlands), and expressed in $\mathrm{ng} / \mathrm{mL}$.

\section{Histopathology}

Lung necropsy specimens were collected from the piglets after euthanasia. Slices of lung tissue were fixed in $10 \%$ buffered form- sham animal (d) are displayed. HE. c Alveolar infiltration with neutrophils, increased occurrence of alveolar macrophages, alveolar capillary congestion, and intra-alveolar edema were evident in meconium-exposed lung tissue. $\mathbf{d}$ No evident lesions except from varying signs of alveolar capillary congestion were observed in sham lung tissue. Data are presented as mean \pm SEM. $B$, baseline.

aldehyde and embedded in paraffin. Sections (2- to 4- $\mu$ m-thick) were stained with hematoxylin and eosin, periodic acid Schiff, Martius scarlet blue, and phosphotungstic acid-hematoxylin stains, and then studied under a Nikon Eclipse 50i light microscope. Photomicrographs were captured with a Nikon DS-U1 digital camera.

\section{Statistics}

GraphPad Prism v7 (San Diego, CA, USA) was used for the statistical analysis. The data were analyzed by an area under the curve (AUC) test and an unpaired $t$ test with equal standard deviation. A $p$ value $<0.05$ was considered statistically significant. 
Fig. 3. The complement inhibitory efficacy of SOBI002. Complement activity was measured in serum in all animals at baseline, at time 0 , and at 240 and $300 \mathrm{~min}$. a Heat-aggregated IgG-coated plates were used to activate complement, and the degree of complement activity was related as percent activity relative to complement activation obtained in a pool of adult pig serum. b Systemic complement activation measured as soluble (s)C5b-9, was evaluated in all animals from baseline and throughout the experiment. Data are presented as mean \pm SEM. B, baseline; CAU, complement arbitrary units.

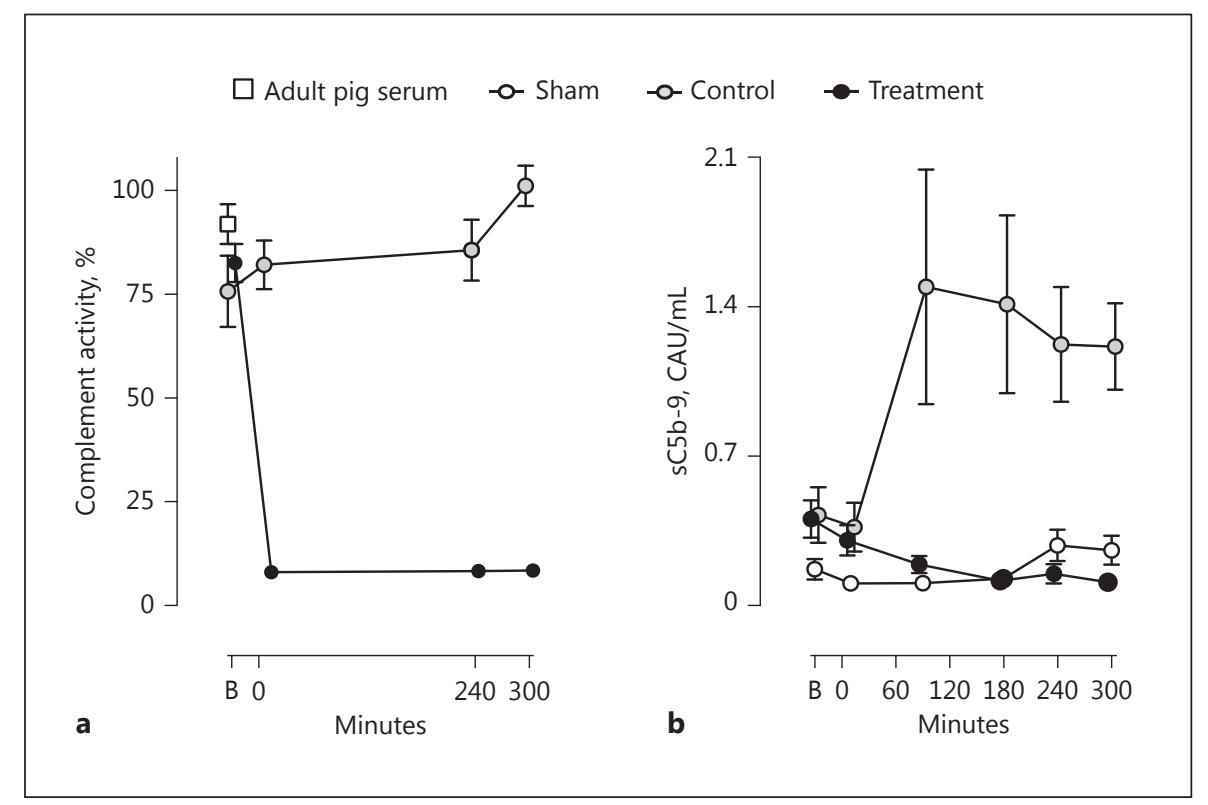

Ethics

The animals were treated in accordance with the Norwegian laboratory animal regulations, by researchers certified by the Federation of European Laboratory Animals Science Association, and the study was approved by the Norwegian Animal Research Authority. Informed written consent was obtained from each human whole blood donor.

\section{Results}

Lung Function and Histopathology in MAS in vivo

Intratracheal meconium instillation induced severe lung injury to all animals included in the control and treatment group. Severe hypoxia, measured by a steep decrease in $\mathrm{PaO}_{2} / \mathrm{FiO}_{2}$-ratio which persisted throughout the study (Fig. 2a), and ventilation index increased similarly, indicating stiff lungs (Fig. 2b). Meconium-exposed lung tissues, irrespective of the intervention, were heavier, firmer, and darker red than the saline-exposed lung tissues, and sank instead of floating when immersed in the formalin solution. Histological examination revealed a massive alveolar infiltration by neutrophils, an increased occurrence of alveolar macrophages, alveolar capillary congestion, intra-alveolar edema, and occasional alveolar hyaline membranes in the meconium-exposed lung tissue (Fig. 2c). No evident lesions except for varying signs of alveolar capillary congestion were present among the sham animals that did not receive any meconium (Fig. 2d).
The Complement Inhibitory Efficacy of SOBI002 in vivo

To ensure complete C5-cleavage inhibition after SOBI002 administration, complement activity was measured in serum from animals included in the study. Heataggregated IgG-coated plates were used to activate complement, and the degree of activation was related as percent activity relative to complement activity in a pool of adult pig serum. SOBI002 completely ablated the generation of sC5b-9 immediately after administration and remained effective throughout the experiment (Fig. 3a). The mean complement activity, including all piglets at baseline, was $82 \%$ (SD 22\%) versus the activity obtained in adult pig serum $(p=0.17)$. The formation of sC5b-9 was also measured in plasma from all animals at baseline and throughout the experiment. In the control group, sC5b-9 increased considerably from time 0 to $90 \mathrm{~min}$, and remained at the same high level thereafter. In the treatment group, SOBI002 attenuated the formation of sC5b-9 completely during the whole observational period, comparable to the low level observed in the sham animals (Fig. 3b).

\section{Systemic Inflammation Reflected by Plasma IL-1 $\beta$ and} IL-6

Intratracheal instillation of meconium induced a systemic inflammatory response measured as the increased plasma formation of both IL-1 $\beta$ (Fig. 4a) and IL-6 (Fig. 4b). The combined inhibition of C5 and CD14 attenuated the formation of IL- $1 \beta$ by $60 \%(p=0.03)$ (Fig. $4 c$ ) and the formation of IL- 6 by $44 \%(p=0.01)$ (Fig. $4 \mathrm{~d}$ ) ver- 
Fig. 4. Systemic inflammation, IL-1 $\beta$, and IL-6. Plasma concentrations of IL-1 $\beta$ (a) and IL-6 (b) were measured at all time points. Statistical comparisons, measured as differences in AUC for IL-1 $\beta$ (c) and IL-6 (d), were performed between animals receiving combined inhibition of $\mathrm{C} 5$ and CD14 versus the nontreated control group. Data are presented as means \pm SEM and as mean $\pm 95 \%$ CI. B, baseline. ${ }^{*} p<0.05$.

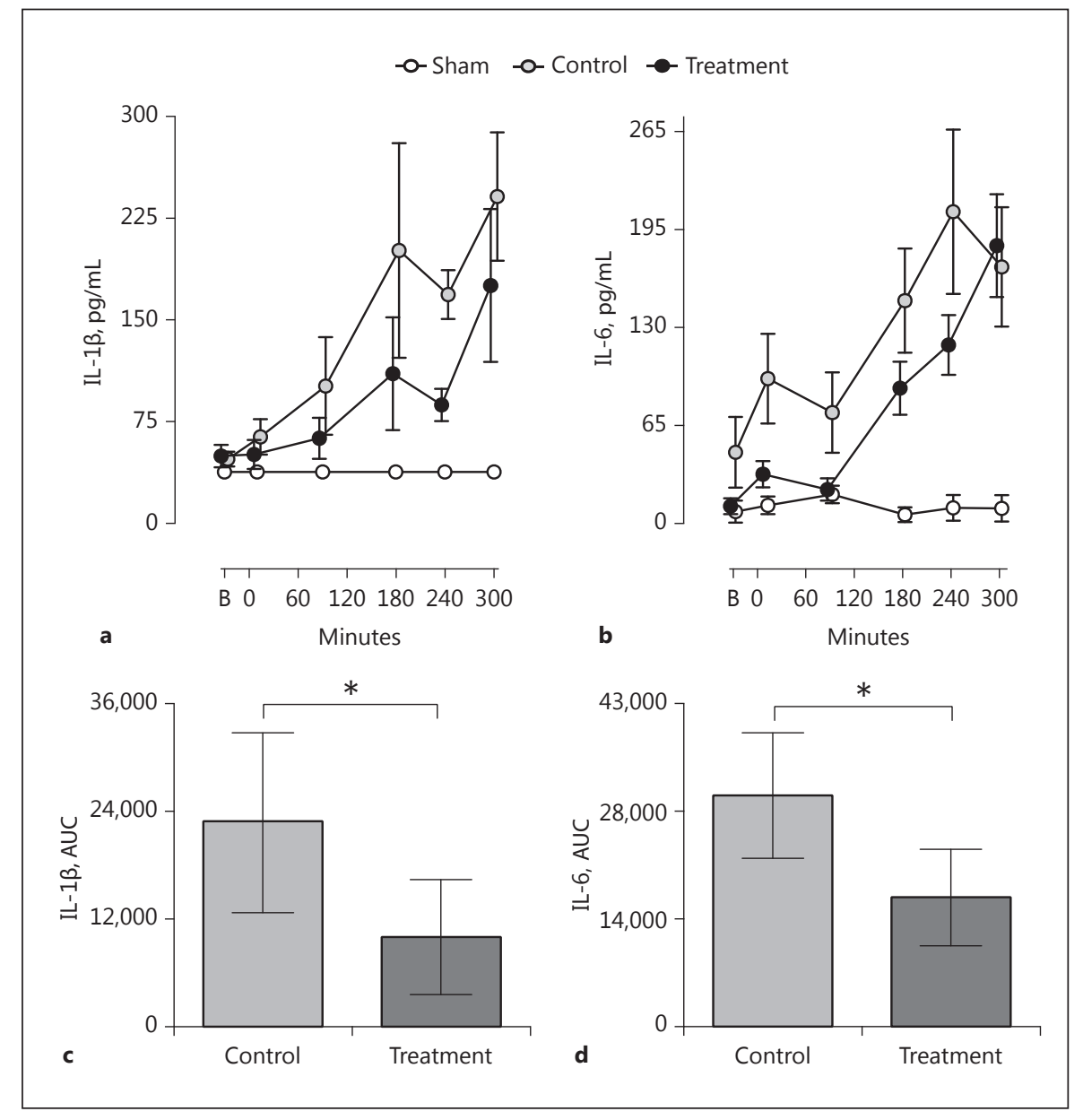

sus the nontreated control group. TNF did not increase after the instillation of meconium whereas IL-8 increased moderately in both groups, but an extensive interindividual measure variation precluded statistical interpretation (data not shown).

\section{$M P O$ Activity in BALF}

The levels of MPO activity measured in the BALF at baseline were similar in all animal groups (Fig. 5a). At the end of the experiment, the treatment group receiving the combined inhibition of $\mathrm{C} 5$ and $\mathrm{CD} 14$ had a $42 \%$ reduction of MPO activity versus the nontreated control group ( $p=0.017)$. Notably, at the end of the experiment, the sham animals had similar MPO activity to that of the treatment group.

\section{Examination of $M P O$ Release ex vivo}

The release of MPO into the BALF prompted us to investigate the role of C5 and CD14 in our standard whole- blood model where we previously showed the pronounced effect on cytokine release ex vivo by inhibiting these molecules [9]. Meconium incubated in human whole blood induced a substantial increase in MPO release within 30 min of incubation (Fig. 5b). Single inhibition with either SOBI002 or anti-CD14 hardly changed meconium-induced MPO release. However, combined inhibition of C5 and CD14 markedly attenuated MPO release by $64 \%$ ( $p=$ 0.008 ), reaching levels lower than the spontaneous background activity observed during incubation, indicating that a substantial synergistic effect was obtained with the combined regimen (Fig. 5b).

\section{Discussion}

This study demonstrates, for the first time, that combined inhibition of C5 and CD14 attenuates the systemic inflammatory response induced by a forceful model of 


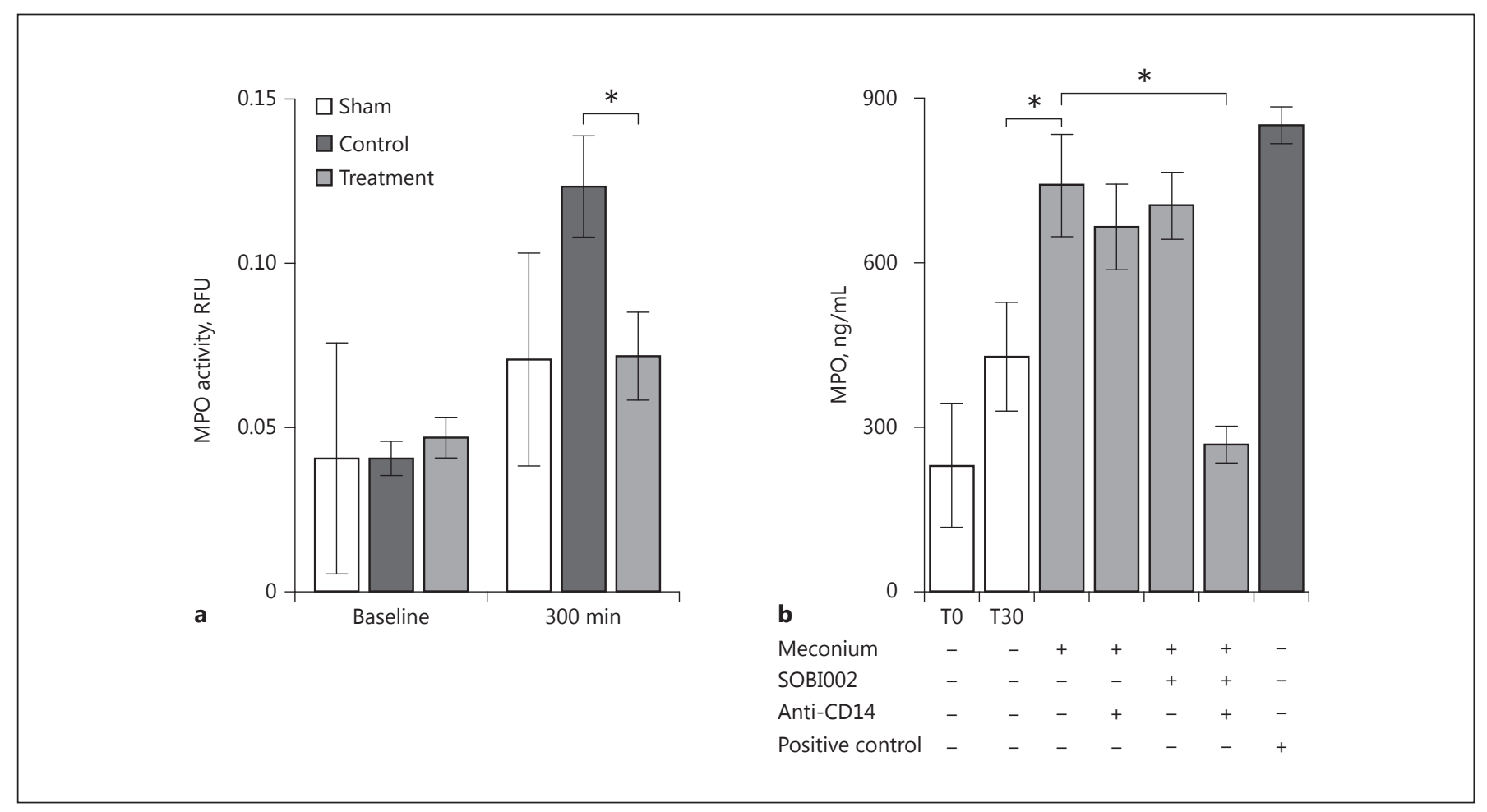

Fig. 5. Myeloperoxidase in bronchoalveolar lavage fluid and in ex vivo human whole blood experiments. MPO-activity was measured in bronchoalveolar lavage fluid at baseline and at the end of the experiment in all animals. a Activity is expressed as relative fluorescence units (RFU) referred to an internal standard provided with the assay. Statistical analysis was performed to compare the treatment with the control group at the end of the experiment, at
300 min. b Meconium-induced inflammation in human whole blood was explored ex vivo, and the inhibitory effect of C5, CD14 and the combination thereof were evaluated. Data are presented as mean \pm SEM. Statistical comparisons were performed between meconium-incubated whole blood and background at $30 \mathrm{~min}$ (T30), and between meconium-incubated whole blood and the effect of the combined inhibition of C5 and CD14. ${ }^{*} p<0.05$.
MAS in piglets. In particular, the inflammasome-related IL-1 $\beta$ cytokine was substantially inhibited. Additionally, MPO activity in the lungs was significantly attenuated by the combined regimen, indicating attenuated neutrophilmediated tissue injury. The combined regimen's effect on meconium-induced MPO release in human whole blood clearly revealed a pronounced and synergistic attenuating effect, with a negligible effect being obtained by the single inhibition of either C5 or CD14.

In this study, meconium instillation caused an excessive local inflammatory reaction, evidenced by the copious infiltration of neutrophils, the degradation of tissue architecture, and the induced wet and stiff lungs. Meconium is a potent activator of neutrophils and alveolar macrophages known to promote fatal lung injury by the liberation of inflammatory mediators and oxygen radicals [19]. Thus, it is reasonable to suggest that the profuse local inflammatory reaction was the primary cause of the observed systemic inflammatory response with increased formation of sC5b-9, IL- $1 \beta$, and IL- 6 . This is in agreement with observations in neonatal patients [20].

The inhibitory effect of the combined regimen on IL$1 \beta$ might be of inflammatory relevance as systemic IL- $1 \beta$ formation is activated by caspase- 1 in the inflammasome, triggering numerous inflammatory responses [21]. Complement activation has been linked to inflammasome activation, and the combination of C5a and TNF has been demonstrated to be an effective primer of the NLRP3 inflammasome [22].

IL-6, a pleiotropic proinflammatory cytokine was efficiently attenuated by the combined inhibition of $\mathrm{C} 5$ and CD14. CD14 is a promiscuous upstream molecule implicated in numerous downstream inflammatory effects. Previous reports of bacteria- and meconium-induced inflammation clearly demonstrate that IL-6 is highly CD14dependent whereas IL-1 $\beta$ has been shown to be both CD14- and complement-dependent [10, 23]. Although these immune pathways act partly independent, they are 
mutually connected by considerable cross-talk, implying that a combined inhibition of the pathways inherit a pronounced anti-inflammatory potential for exogenously as well as endogenously induced inflammation [11]. The complement inhibitor used in this study, SOBI002, efficiently and completely prevented systemic sC5b-9 formation, and thus also the formation of the potent anaphylatoxin C5a. Interestingly, the complement activity measured in the piglets in this cohort at baseline was not significantly lower than in adult pigs, reflecting that these young animals displayed a surprisingly mature and competent complement system.

The peroxidase enzyme MPO is released upon oxidative burst, is prone to cause damage to host cells and tissue, and is implicated in inflammatory lung pathologies [19]. The combined inhibition of C5 and CD14 attenuated MPO activity in the BALF by $42 \%$. However, it is not possible to differentially attribute the specific effect achieved by inhibiting the 2 pathways, so ex vivo experiments using human whole blood were performed. Individual C5 or CD14 inhibition did not attenuate meconium-induced MPO formation in human whole blood, but inhibition of the 2 in combination significantly attenuated the formation by $64 \%$. Similar observations have previously been made in bacteria-induced inflammation and reflect a synergistic interaction between the complement system and CD14 [24]. This phenomenon underscores the redundancy of host defense where one of the pathways substitutes the other if it is blocked.

The study focused on the inflammatory impact of MAS and specifically addressed the role of the upstream part of the innate immune system. Complement, with the central C5 molecule, and the TLR system, with the important coreceptor CD14, were particularly addressed in this study. Although clinically less relevant than a delayed intervention, the inhibitors were provided prior to meconium instillation, thus minimizing an initial activation of these upstream innate immune pathways, as a proof of concept design. The high concentration of meconium used rapidly induced respiratory distress in all the animals and so they required extensive respiratory support. However, no improved lung function was observed by the combined treatment regimen, and it is reasonable to suggest that beneficial effects may have been masked by the overwhelming severity of the model. Still, the model induced a systemic inflammatory response in all animals challenged with meconium, underscoring the important role of inflammation in MAS. Importantly, the data demonstrate clearly that combined inhibition of C5 and CD14 had a pronounced attenuating effect on the systemic release of deleterious inflammatory mediators (particularly IL-1 $\beta$ ), indicating inflammasome activation but also IL- 6 and local MPO activity in the BALF.

These novel findings should be considered in studies exploring future therapeutic approaches for the treatment of MAS.

\section{Disclosure Statement}

The authors declare no conflicts of interest.

\section{References}

1 Haakonsen Lindenskov PH, Castellheim A, Saugstad OD, Mollnes TE: Meconium aspiration syndrome: possible pathophysiological mechanisms and future potential therapies. Neonatology 2015;107:225-230.

2 Goldsmith JP: Continuous positive airway pressure and conventional mechanical ventilation in the treatment of meconium aspiration syndrome. J Perinatol 2008;28(suppl 3): S49-S55.

3 Anwar Z, Butt TK, Kazi MY: Mortality in meconium aspiration syndrome in hospitalized babies. J Coll Physicians Surg Pak 2011;21: 695-699.

4 de Beaufort AJ, Bakker AC, van Tol MJ, Poorthuis BJ, Schrama AJ, Berger HM: Meconium is a source of pro-inflammatory substances and can induce cytokine production in cultured A549 epithelial cells. Pediatr Res 2003; 54:491-495.
5 Zagariya A, Sierzputovska M, Navale S, Vidyasagar D: Role of meconium and hypoxia in meconium aspiration-induced lung injury in neonatal rabbits. Mediat Inflamm 2010;2010:204831.

6 Castellheim A, Lindenskov PHH, Pharo A, Aamodt G, Saugstad OD, Mollnes TE: Meconium aspiration syndrome induces complement-associated systemic inflammatory response in newborn piglets. Scand J Immunol 2005;61:217-225.

7 Hofer N, Jank K, Strenger V, Pansy J, Resch B: Inflammatory indices in meconium aspiration syndrome. Pediatr Pulmonol 2016;51: 601-606.

8 Lindenskov PHH, Castellheim A, Aamodt G, Saugstad OD, Mollnes TE: Complement activation reflects severity of meconium aspiration syndrome in newborn pigs. Pediatr Res 2004;56:810-817.
9 Salvesen B, Fung M, Saugstad OD, Mollnes TE: Role of complement and CD14 in meconium-induced cytokine formation. Pediatrics 2008;121:e496-e505.

10 Salvesen B, Stenvik J, Rossetti C, Saugstad OD, Espevik T, Mollnes TE: Meconium-induced release of cytokines is mediated by the TRL4/MD-2 complex in a CD14-dependent manner. Mol Immunol 2010;47:1226-1234.

11 Barratt-Due A, Pischke SE, Nilsson PH, Espevik T, Mollnes TE: Dual inhibition of complement and Toll-like receptors as a novel approach to treat inflammatory diseases- $\mathrm{C} 3$ or C5 emerge together with CD14 as promising targets. J Leukoc Biol 2017;101:193-204.

12 Tollofsrud PA, Solas AB, Saugstad OD: Newborn piglets with meconium aspiration resuscitated with room air or $100 \%$ oxygen. Pediatr Res 2001;50:423-429. 
13 Morgan BP, Harris CL: Complement, a target for therapy in inflammatory and degenerative diseases. Nat Rev Drug Discov 2015;14:857877.

14 Lau C, Gunnarsen KS, Hoydahl LS, Andersen JT, Berntzen G, Pharo A, Lindstad JK, Ludviksen JK, Brekke OL, Barratt-Due A, Nielsen EW, Stokes CR, Espevik T, Sandlie I, Mollnes TE: Chimeric anti-CD14 IGG2/4 hybrid antibodies for therapeutic intervention in pig and human models of inflammation. J Immunol 2013;191:4769-4777.

15 Lindenskov PHH, Castellheim A, Aamodt G, Saugstad OD: Meconium-induced IL-8 production and intratracheal albumin alleviated lung injury in newborn pigs. Pediatr Res 2005; 57:371-377.

16 Mollnes TE, Brekke O-L, Fung M, Fure H, Christiansen D, Bergseth G, Videm V, Lappegård KT, Köhl J, Lambris JD: Essential role of the $\mathrm{C} 5 \mathrm{a}$ receptor in $\mathrm{E}$. coli-induced oxidative burst and phagocytosis revealed by a novel lepirudin-based human whole blood model of inflammation. Blood 2002;100: 1869-1877.
17 Bergseth G, Ludviksen JK, Kirschfink M, Giclas PC, Nilsson B, Mollnes TE: An international serum standard for application in assays to detect human complement activation products. Mol Immunol 2013;56:232-239.

18 Mollnes TE, Lea T, Froland SS, Harboe M: Quantification of the terminal complement complex in human plasma by an enzymelinked immunosorbent assay based on monoclonal antibodies against a neoantigen of the complex. Scand J Immunol 1985;22:197-202.

19 Klebanoff SJ: Myeloperoxidase: friend and foe. J Leukoc Biol 2005;77:598-625.

20 Okazaki K, Kondo M, Kato M, Kakinuma R, Nishida A, Noda M, Taniguchi K, Kimura H: Serum cytokine and chemokine profiles in neonates with meconium aspiration syndrome. Pediatrics 2008;121:e748-e753.

21 Horvath GL, Schrum JE, De Nardo CM, Latz E: Intracellular sensing of microbes and danger signals by the inflammasomes. Immunol Rev 2011;243:119-135.
22 Samstad EO, Niyonzima N, Nymo S, Aune MH, Ryan L, Bakke SS, Lappegard KT, Brekke OL, Lambris JD, Damas JK, Latz E, Mollnes TE, Espevik T: Cholesterol crystals induce complement-dependent inflammasome activation and cytokine release. J Immunol 2014; 192:2837-2845.

23 Lappegard KT, Christiansen D, Pharo A, Thorgersen EB, Hellerud BC, Lindstad J, Nielsen EW, Bergseth G, Fadnes D, Abrahamsen TG, Hoiby EA, Schejbel L, Garred P, Lambris JD, Harboe M, Mollnes TE: Human genetic deficiencies reveal the roles of complement in the inflammatory network: lessons from nature. Proc Natl Acad Sci USA 2009;106:15861-15866.

24 Egge KH, Thorgersen EB, Lindstad JK, Pharo A, Lambris JD, Barratt-Due A, Mollnes TE: Post-challenge inhibition of $\mathrm{C} 3$ and $\mathrm{CD} 14$ attenuates Escherichia coli-induced inflammation in human whole blood. Innate Immun 2014;20:68-77 